Ann. Biol. anim. Bioch. Biophys., I963, 3 (3), 307-323

\title{
ÉTUDE CRITIQUE DU DOSAGE DE ICSH PAR LA MÉTHODE DE L'ACIDE ASCORBIQUE OVARIEN
}

\author{
J. PELLETIER \\ avec la collaboration technique de Marie-Madeleine de KEviers et Christine VIÉ. \\ Station centrale de Physiologie animale, \\ Centre national de Recherches zootechniques, Jouy-en-Josas (Seine-et-Oise)
}

\section{SOMMAIRE}

L'étude du dosage de ICSH par la technique de PARLow a montré que pour des rats appartenant à la race Wistar des modifications dans le prétraitement des animaux augmentent considérablement la précision de la méthode.

Ces modifications comprennent :

- une diminution de la dose de PMSG (3o U.I.),

- un intervalle de temps plus important entre les injections de PMSG et HCG (7o h),

- l'utilisation d'animaux plus jeunes lors du début du prétraitement (23 j).

En outre la précision est augmentée par l'emploi d'un intervalle de 3 heures entre l'injection de ICSH et l'abattage des animaux et par la sélection génétique à l'intérieur de la race Wistar.

\section{INTRODUCTION}

La méthode de dosage de ICSH proposée par PARLOW (I958) et basée sur la mesure de la diminution de l'acide ascorbique ovarien, repose sur les observations de ClaEsson (I949) et de KARG (I957) montrant que les hormones gonadotropes produisent une chute de la teneur en acide ascorbique de l'ovaire de la ratte adulte.

La sensibilité et la spécificité de cette technique, montrée par Mc CaNn et TALEISNIK (I960), 1'ont fait adopter par la majorité des auteurs. Toutefois la précision de la méthode s'est révélée très variable suivant les utilisateurs et semble dépendre de la race des animaux-test employés. En particulier des résultats très médiocres sont obtenus avec des rattes de la race Wistar où la droite de régression de l'acide ascorbique en fonction de la dose de ICSH a une pente faible et inconstante, se 
traduisant fréquemment par un non-parallélisme entre les courbes de réponse des préparations standard et inconnue (SCHMID'T-EI,MENDORFF et LORAINE, I962; HuTCHINSON, WoL,THUIs, communications personnelles).

Les différentes souches d'animaux utilisés dans ce laboratoire (race Wistar) ont montré des sensibilités extrêmement différentes à l'hormone FSH, il nous a semblé intétessant d'étudier leur comportement au dosage d'ICSH par la technique de PARLOW, et à cette fin de rechercher les modifications éventuelles à apporter au protocole initialement proposé.

En effet, constatant chez ces souches de rats, que le prétraitement ne produisait qu'un faible développement de l'ovaire, nous avons cherché à augmenter celui-ci et en conséquence, la teneur totale en acide ascorbique. A cet effet un certain nombre de facteurs résultant du principe même du dosage ont été étudiés :

- l'influence de la dose de PMSG sur le poids et l'acide ascorbique ovariens ;

- l'influence de l'intervalle entre les 2 injections, PMSG d'une part et HCG d'autre part;

- l'influence de la dose de HCG ;

- l'influence de l'âge des corps jaunes ;

- l'influence de l'âge des animaux.

Cette étude du prétraitement des animaux-test a été suivie d'une seconde portant plus particulièrement sur le dosage de ICSH lui-même :

$\mathrm{ICSH}$

- cinétique de la quantité de la vitamine $\mathrm{C}$ dans l'ovaire après injection de

— étude de la courbe de réponse de l'acide ascorbique ovarien à différentes doses d'hormone interstitielle stimulante;

- influence génétique sur la réponse de l'acide ascorbique ovarien après injection de ICSH.

\section{MATÉRIEI, ET MÉTHODES}

$\left.I^{0}\right)$ Choix des animaux. -- Les animaux utilisés pour le dosage biologique sont des rattes immatures de race Wistar appartenant aux souches développées à Jouy-en-Josas et numérotées 03, o4 et I2. La première est utilisée pour l'ensemble des expériences, les deux autres pour l'étude, par comparaison, de l'influence génétique sur la précision du dosage. Tous les animaux sont sevrés à l'âge de 23 jours et reçoivent alors une alimentation standardisée.

$\left.2^{\circ}\right)$ Produits employés. - Pour le prétraitement, l'hormone sérique Gestyl (PMSGOrganon) et l'hormone chorionique Pregnyl (HCG Organon) ont été utilisées. Une partie de cette étude a été effectuée par référence avec le standard International HCG. Ces hormones sont administrées par injection sous-cutanée après dissolution dans $0,5 \mathrm{cc}$ de sérum physiologique.

Pour l'établissement des courbes de diminution de l'acide ascorbique dans l'ovaire par l'hormone interstitielle stimulante, une préparation purifiée d'hypophyse de Mouton a été employée (LH-NIH, lot $n^{0}$ I I 805). L'administration est effectuée dans $0,25 \mathrm{cc}$ de sérum physiologique par voie intraveineuse.

Les produits chimiques, acide I-ascorbique et dichlorophenolindophen ont été choisis purs, et cristallisés (produits Eastman).

$\left.3^{\circ}\right)$ Technique de dosage de ICSH. - L'administration de l'hormone standard LH-NIH est effectuée par injection dans la veine jugulaire gauche en passant au travers des muscles pectoraux.

Sous anesthésie à l'éther, un ovaire contrôle est prélevé avant l'injection, puis, à un intervalle de temps fixé après celle-ci, les animaux sont abattus par fracture de la colonne vertébrale au niveau des vertèbres cervicales, le retrait du deuxième ovaire est alors effectué. 
Dès le prélèvement, l'ovaire est séparé de l'oviducte et de la capsule ovarienne et pesé, il est alors mis dans de l'acide métaphosphorique à 2,5 p. 10o pour le dosage de l'acide ascorbique. Celui-ci est effectué selon la technique de Minduin et BUTLER (I937) modifiée par Munson (I955) utilisant la décoloration de dichlorophenolindophenol.

$\left.4^{\circ}\right)$ Techniques histologiques. - Les ovaires de rattes prélevés pour l'étude histologique ont été fixés 48 heures au Bouin Hollande et inclus dans la paraffine. Les coupes ont été effectuées à ro $\mu$ et colorées par l'hématoxyline éosine ou le trichrome de Masson.

$\left.5^{\circ}\right)$ Analyse statistique des résultats. - Les résultats ont été exprimés en teneur totale de l'ovaire en vitamine $\mathrm{C}$, la moyenne d'un lot expérimental pouvant être ajustée après correction du poids ovarien par analyse de covariance.

La détermination de la signification statistique a été effectuée soit par le test $t$ de STUDENT en comparant les différents niveaux expérimentaux à l'un d'eux pris comme référence, soit par analyse de covariance lorsque le poids ovarien peut être considéré comme variable indépendante du traitement.

Les dosages biologiques ont été des dosages en 4 points, l'analyse étant effectuée selon EMMENs (1948) et BLISS (1951).

\section{RÉSULTATS}

\section{INFLUENCE DE DIFFÉRENTS FACTEURS SUR LE POIDS OVARIEN E'T LA TENEUR EN ACIDE ASCORBIQUE}

\section{Io) Influence de la dose d'hormone sérique}

Selon le protocole A défini dans le tableau I des doses variant de 5 U.I. 70 U.I. de PMSG sont administrées à des rattes âgées de 25 jours en une seule injection. $5^{6}$ heures plus tard les animaux reçoivent 25 U.I. de HCG, les animaux étant abattus après un nouveau délai de cinq jours et demi.

\section{a) Influence de PMSG sur le poids ovarien.}

Le tableau 2 montre que le poids ovarien augmente d'une façon considérable pour des doses croissant de 5 à 30 U.I. d'hormone sérique. De 30 à 70 U.I. il n'y a plus d'augmentation du poids ovarien (graph. I).

\section{b) Infuence de PMSG sur l'acide ascorbique ovarien.}

Les résultats sont ici différents de ceux concernant les poids des ovaires : en effet, la quantité d'acide ascorbique passe par un maximum pour la dose de 30 U.I. de PMSG (graph. 2). La différence entre les doses de 30 U.I. et 40 U.I. n'est pas significative, alors qu'une forte diminution se manifeste pour 50 U.I. et 70 U.I. de PMSG.

L'étude histologique des ovaires révèle que leur lutéinisation après l'injection d'une dose d'hormone sérique de 70 U.I. est imparfaite. En revanche, la diminution de la dose injectée à 50 U.I., puis à 30 U.I. montre une amélioration de la lutéinisation qui, pour cette dernière dose, est pratiquement totale, le volume relatif du tissu lutéinique étant rarement inférieur à 80 p. Ioo.

L'ensemble de ces résultats nous a conduit à adopter la dose de 30 U.I. de PMSG pour le prétraitement des animaux dans la suite de cette étude. 


\section{TABLEAU I}

Protocoles utilisés pour l'étude des différents facteurs influençant la technique de dosage de ICSH par la détermination de l'acide ascorbique ovarien

\begin{tabular}{|c|c|c|c|c|c|}
\hline Protocole utilisé & $\underset{\text { (jours) }}{\text { Age des anim. }}$ & $\begin{array}{l}\text { Dose PMSG } \\
\text { (U.I.) }\end{array}$ & $\begin{array}{c}\text { Dose HCG } \\
\text { (U.I.) } \\
\text { (heures) }\end{array}$ & $\begin{array}{c}\text { Intervalle } \\
\text { PMSG-HCG }\end{array}$ & $\begin{array}{c}\text { Abattage } \\
\text { après HCG } \\
\text { (jours) }\end{array}$ \\
\hline 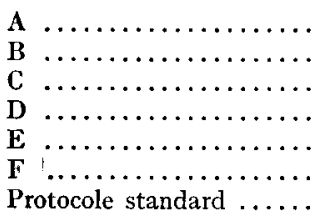 & $\begin{array}{c}25 \\
25 \\
23 \\
25 \\
25 \\
21 \text { à } 33 \\
23\end{array}$ & $\begin{array}{l}5 \text { à } 70 \\
30 \\
40 \\
30 \\
30 \\
30 \\
30\end{array}$ & $\begin{array}{c}25 \\
25 \\
25 \\
18,5 \stackrel{\text { à }}{75} \\
25 \\
25 \\
25\end{array}$ & $\begin{array}{l}\quad 62 \\
48 \stackrel{\text { à }}{94} \\
46 \stackrel{\text { a }}{118} \\
\quad 70 \\
70 \\
70 \\
70\end{array}$ & 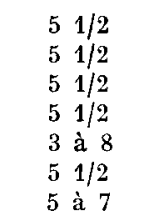 \\
\hline
\end{tabular}

\section{TABIEAU 2}

Infuence de la dose de PMSG sur le poids d'un ovaire $(X)$, et sur la quantité d'acide ascorbique de cet ovaire $(Y)$

\begin{tabular}{|c|c|c|c|}
\hline $\begin{array}{c}\text { Dose } \\
\text { (U. I.) }\end{array}$ & $\begin{array}{l}\text { Nbre de } \\
\text { données }\end{array}$ & $\overline{\mathrm{X}} \underset{(\mathrm{mg})}{ \pm S m}$ & $\overline{\bar{Y}} \underset{(\mu g)}{ \pm S m}$ \\
\hline 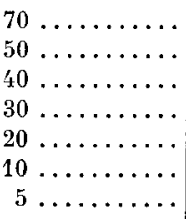 & $\begin{array}{l}42 \\
34 \\
30 \\
64 \\
44 \\
34 \\
34\end{array}$ & $\left|\begin{array}{l}77,1 \pm 1,47 \\
77,6 \pm 2,84 \\
77,3 \pm 1,42 \\
77,8 \pm 2,01 \\
67,1 \pm 2,67\left(^{(2}\right) \\
22,9 \pm 0,64\left(^{(2}\right) \\
14,8 \pm 0,43\left(^{2}\right)\end{array}\right|$ & $\begin{array}{l}63,1 \pm 1,85\left(^{2}\right) \\
67,3 \pm 3,25\left(^{2}\right) \\
77,7 \pm 2,14 \\
80,8 \pm 1,62 \\
68,1 \pm 3,16\left(^{2}\right) \\
13,6 \pm 1,20\left(^{2}\right) \\
6,2 \pm 0,50\left(^{2}\right)\end{array}$ \\
\hline
\end{tabular}

(2) Différent de la moyenne obtenue pour 30 U.I. PMSG, $\mathbf{P}<0,01$.

$\left.2^{\circ}\right)$ Infuence de l'intervalle entre les injections de PMSG et HCG sur le poids de l'ovaire et l'acide ascorbique

L'injection de l'hormone sérique entraîne donc une stimulation folliculaire importante et il est naturel de penser que l'état de développement des follicules est fonction du temps s'écoulant après l'injection de PMSG.

L'influence de l'intervalle de temps entre les injections d'hormones sérique (3o U.I.) et chorionique (25 U.I.) a été déterminée sur des lots d'animaux âgés de 25 jours pour lesquels ce facteur variait de 48 à 94 heures (expérience A, protocole B, tableau I). 


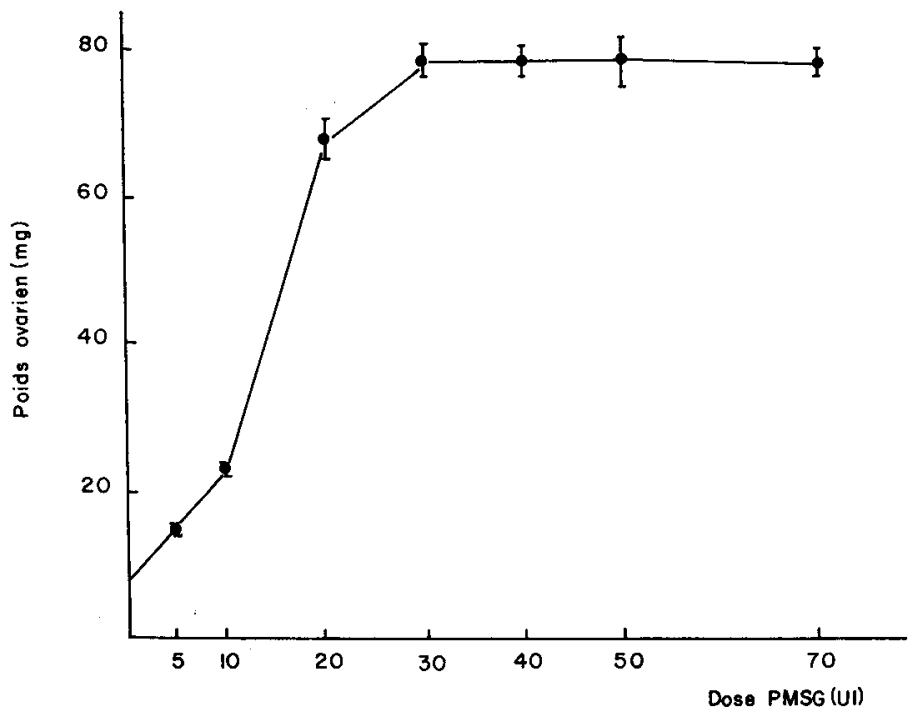

GRAPHIQUe I. - - Influence de la dose de PMSG injectée à des raltes de 25 jours sur le poids d'un ovaire après lutéinisation obtenue par injection de $25 \mathrm{U}$. I. de HCG, 62 heures plus tard. (Intervalle entre l'injection de $H C G$ et l'abattage des animaux : 5 iours et demi.)

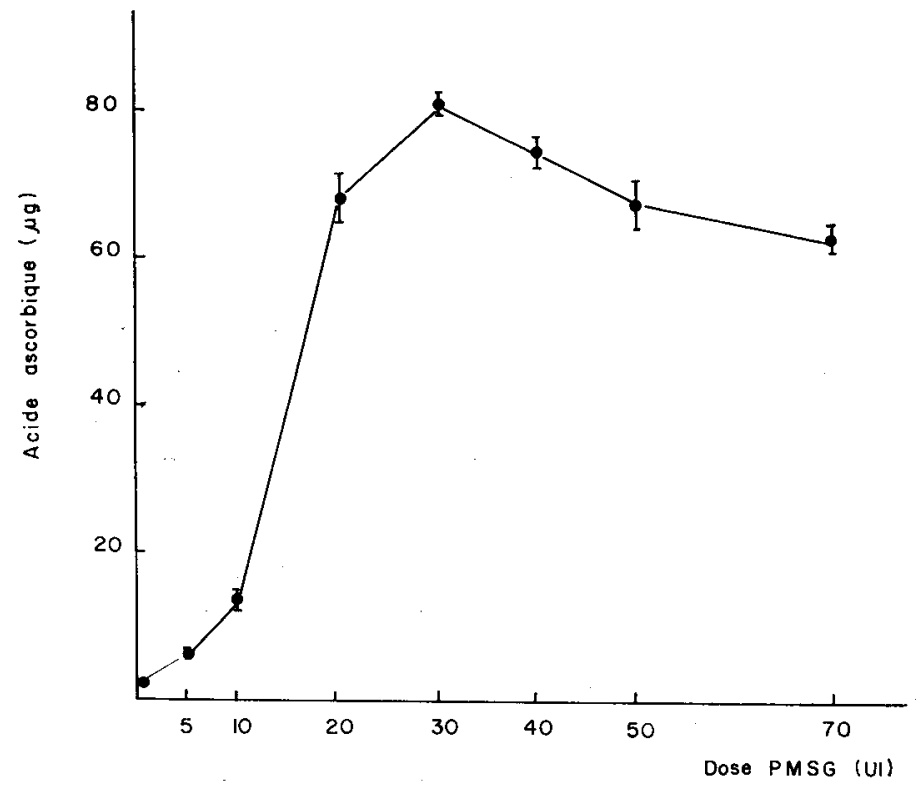

Graphique 2. - Infuence de la dose de PMSG injectée des rattes de 25 jours sur la quantité d'acide ascorbique contenue dans un ovaire atrès lutéinisation obtenue par injection de $25 \mathrm{U}$. I. de HCG, 62 heures plus tard. (Intervalle entre l'injection de HCG et l'abattage des animaux 5 jours et demi.) 
a) Infuence de l'intervalle PMSG-HCG sur le poids ovarien.

Le tableau 3 et le graphique 3 montrent que les intervalles les plus faibles de 48 à 55 heures ne permettent pas une croissance maximum de l'ovaire. Aux intervalles de 62 et 70 heures correspond un développement ovarien équivalent entre eux mais plus important que précédemment ; par la suite, le poids ovarien croît encore, mais d'une façon irrégulière qui se manifeste par une variabilité plus forte.

TABLEAU 3

Influence de l'intervalle entre l'injection de PMSG et celle de HCG sur le poids d'un ovaire $(X)$ et sur la quantité d'acide ascorbique de cet ovaire $(Y)$

\begin{tabular}{|c|c|c|c|c|}
\hline Intervalle (heures) & Nbre de données & $\bar{X} \pm \operatorname{Sm}(\mathrm{mg})$ & $\bar{Y} \pm \operatorname{Sm}(\mu g)$ & $\widehat{V}(\mu g)$ \\
\hline 48 & 26 & $72,05 \pm 2,63\left(^{2}\right)$ & $6,2,42 \pm 2,96\left(^{2}\right)$ & \\
\hline 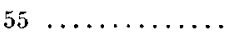 & 24 & $81,96 \pm 2,96$ & $74,33 \pm 3,05\left(^{1}\right)$ & 82,37 \\
\hline$\ldots \ldots \ldots$ & 46 & $88,58 \pm 3,15$ & $81,98 \pm 3,35$ & 83,14 \\
\hline $70 \ldots \ldots \ldots \ldots$ & 43 & $89,73 \pm 2,45$ & $84,29 \pm 3,15$ & 84,26 \\
\hline 78 & 40 & $96,83 \pm 3,15$ & $94,66 \pm 4,10\left(^{1}\right)$ & 87,25 \\
\hline $86 \ldots$ & 15 & $108,17 \pm 4,23\left({ }^{2}\right)$ & $111,93 \pm 5,24(2)$ & \\
\hline $94 \ldots \ldots \ldots \ldots \ldots$ & 18 & $97,1 / 1 \pm 3,95$ & $93,97 \pm 4,48$ & 86,24 \\
\hline
\end{tabular}

$\widehat{\mathrm{Y}}$ acide ascorbique ovarien corrigé par le poids ovarien dans l'intervalle où celui-ci est constant.

(1) Différent de la moyenne obtenue à 70 heures $(\mathrm{P}<0,05)$.

(2) Différent de la moyenne obtenue à 70 heures $(P<0,01)$.

b) Influence de l'intervalle PMSG-HCG sur l'acide ascorbique ovarien.

La quantité d'acide ascorbique suit assez fidèlement les variations des poids ovariens et les mêmes conclusions peuvent approximativement être formulées.

c) Expérience effectuée dans les conditions standard (Expérience B).

L'expérience précédente a été reprise suivant le protocole $\mathrm{C}$ (tableau I) qui diffère du précédent par l'âge plus précoce des animaux ( 23 jours) et la dose de l'hormone sérique (40 U.I.) qui a été adoptée pour cette expérience après vérification de l'activité du lot de PMSG, activité qui s'est révélée légèrement inférieure à celle du lot précédent. En outre l'intervalle de temps entre PMSG et HCG variait de 46 à II 8 heures. Les variations de poids ovarien (graph. 3) indiquent des résultats analogues aux précédents. En particulier, un intervalle de 70 heures entre les injections d'hormone sérique et chorionique est favorable. En outre, pour la commodité de la technique de routine, cet intervalle peut être augmenté jusqu'à 78 heures, sans modifier l'action du prétraitement sur l'ovaire, il doit toutefois être conservé constant. 


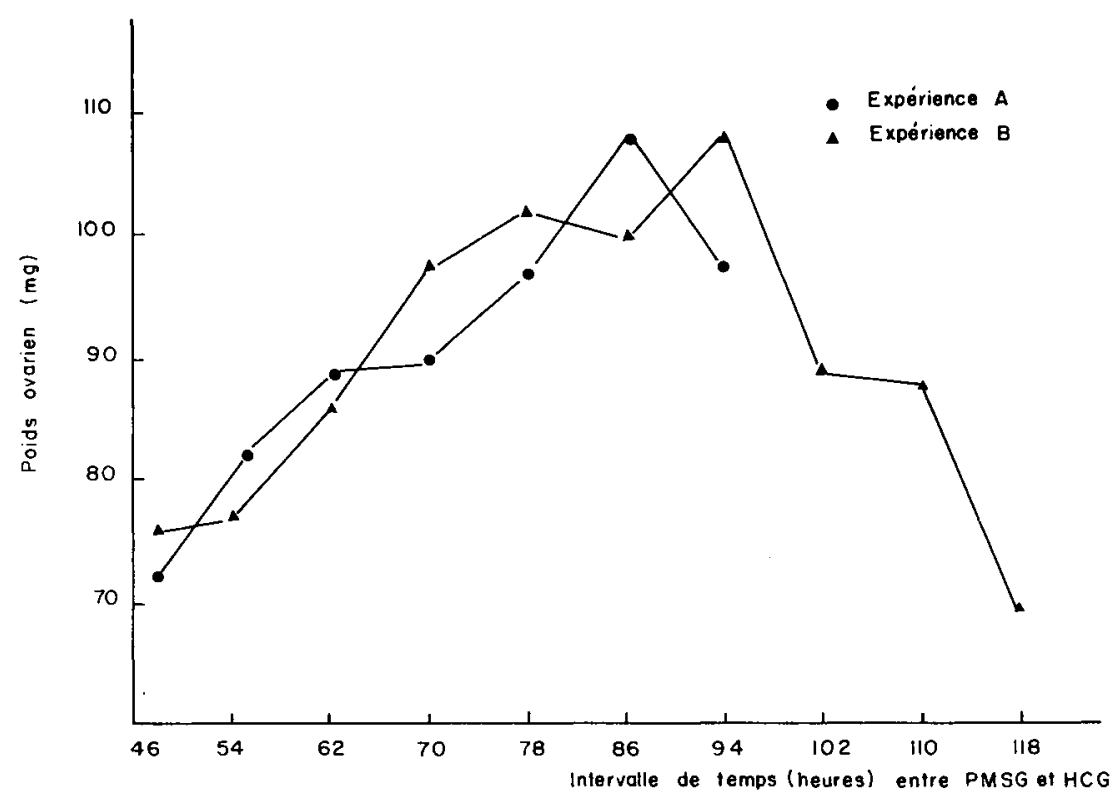

Graphique 3. - Variation du poids d'un ovaire en fonction de l'intervalle de temps séparant l'injection de $P M S G$ de celle de $H C G$

Expérience A (PMSG 30 U.I., HCG 25 U.I., âge des animaux 25 jours).

Expérience B (PMSG 40 U.I., HCG 25 U.I., âge des animaux 23 jours).

(Intervalle HCG-abattage des animaux 5 jours $\mathrm{i} / 2$ dans les deux expériences.)

\section{$\left.3^{\circ}\right)$ Infuence de la dose de HCG sur le poids de l'ovaire et l'acide ascorbique ovarien}

Des rattes traitées par 30 U.I. PMSG ont reçu 70 heures plus tard des doses d'hormone chorionique variant de $I 2,5$ à 75 U.I. en une ou deux injections effectuées à 24 heures d'intervalle (Protocole $\mathrm{D}$, tableau I).

a) Influence de la dose de HCG sur le poids de l'ovaire.

La dose de HCG ne semble pas provoquer une variation importante du poids de l'ovaire que cette dose soit administrée en une ou deux fois (tableau 4).

b) Infuence de la dose de HCG sur la quantité d'acide ascorbique ovarien.

On constate immédiatement que la quantité d'acide ascorbique ovarien est significativement plus faible lorsque la dose de $\mathrm{HCG}$ est de 12,5 U.I. En revanche des doses égales ou supérieures à 25 U.I. ne modifient pas la quantité d'acide ascorbique ovarien.

Par ailleurs l'administration de l'hormone chorionique en 2 fois semble permettre d'obtenir une amélioration de la quantité de vitamine $C$, mais celle-ci n'est pas significative.

C'est pourquoi la dose de 25 U.I. de HCG injectée en une seule fois semble satisfaisante et a été conservée dans le protocole de la technique de dosage.

Anuales de Biologie Animale. - I 963. 
TABIEAT 4

Influence de la dose de $H C G$ sur le poids d'un ovaire $(X)$ et la quantité d'acide ascorbique de cet ovaire $(Y)$

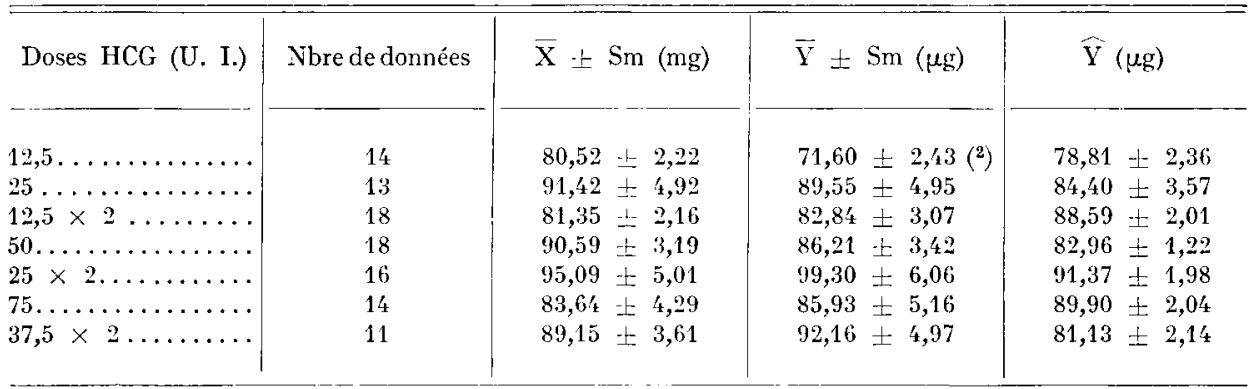

(2) Différent de la moyenne obtenue pour 25 U.I. $\mathrm{HCG}(\mathrm{P}<0,01) \widehat{\mathrm{Y}}$ quantité d'acide ascorbique ovarien corrigé par covariance.

$\left.4^{\circ}\right)$ Infuence de l'âge du corps jaune sur la quantité d'acide ascorbique de l'ovaire

Des rattes âgées de 25 jours ont reçu une injection de 30 U.I. de PMSG suivie 70 heures plus tard par une injection de $\mathrm{HCG}$. L'âge de ces corps jaunes a été confondu avec l'intervalle de temps séparant l'injection de $\mathrm{HCG}$ et le moment de l'abattage effectué 3 à 8 jours après la dernière injection. En réalité, il s'agit d'une approximation puisque l'ovulation ne se produit que plusieurs heures après l'injection de HCG (Protocole E, tableau I).

a) Influence de l'âge des corps jaunes sur le poids ovarien (tableau 5).

Dès le $3^{\mathrm{e}}$ jour les corps jaunes ont atteint leur maximum de développement, les poids ovariens ne sont pas significativement différents les uns des autres entre le $3^{\mathrm{e}}$ et le $8^{\mathrm{e}}$ jour de la pseudo-gestation. La réponse apparemment plus forte à 4 jours est due aux poids exceptionnels des ovaires de l'un des animaux.

b) Influence de l'âge du corps jaune sur l'acide ascorbique ovarien.

L'acide ascorbique croît régulièrement dans l'ovaire $\mathrm{du} 3^{\mathrm{e}}$ au $8^{\mathrm{e}}$ jour mais les résultats, ajustés par covariance, montrent l'existence d'un palier bien défini entre le $5^{\mathrm{e}}$ et le $8^{\mathrm{e}}$ jour (graph. 4) qui est favorable aux conditions du dosage. Des expériences préliminaires nous ayant indiqué que l'acide ascorbique commençait à décroître dans les corps jaunes âgés de 9 jours, c'est durant ce palier, du $5^{\mathbf{e}}$ au $7^{\mathrm{e}}$ jour inclus après l'injection de $\mathrm{HCG}$, que les animaux sont utilisés pour les déterminations de ICSH.

\section{$\left.5^{\circ}\right)$ Influence de l'âge des animaux lors de l'injection d'hormone sérique}

$I_{1}$ a réponse ovarienne au prétraitement est conditionnée par l'état de maturité folliculaire au moment de l'injection de l'hormone sérique mais peut-être aussi 
TABLEAU 5

Influence de l'âge du corps jaune sur le poids de l'ovaire $(X)$ et la quantité d'acide ascorbique de cet ovaire $(Y)$

\begin{tabular}{|c|c|c|c|c|}
\hline $\begin{array}{c}\text { Age du corps jaune } \\
\text { (jours) }\end{array}$ & Nbre de données & $\overline{\mathrm{X}} \pm \mathrm{Sm}(\mathrm{mg})$ & $\bar{Y} \pm \operatorname{Sm}(\mu g)$ & $Y(\mu g)$ \\
\hline 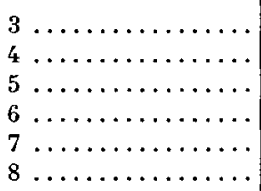 & $\begin{array}{l}15 \\
1 / 4 \\
18 \\
16 \\
18 \\
15\end{array}$ & $\begin{aligned} & 89,8 \pm 6,6^{\prime} \\
& 102,1 \pm 4,40\left.{ }^{2}\right) \\
& 87,1 \pm 3,44 \\
& 84,5 \pm 3,41 \\
& 92,5 \pm 4,65 \\
& 90,6 \pm 4,64\end{aligned}$ & $\begin{array}{r}74,2 \pm 7,15\left(^{2}\right) \\
94,5 \pm 3,44 \\
96,3 \pm 3,62 \\
96,9 \pm 4,82 \\
101,1 \pm 5,38 \\
110,5 \pm 5,15\end{array}$ & $\begin{aligned} & 75,2 \pm 1,70\left(^{(2)}\right. \\
& 82,9 \pm 2,60\left(^{(2)}\right. \\
& 100,1 \pm 1,10 \\
& 103,4 \pm 1,90 \\
& 99,3 \pm 1,26 \\
& 110,7 \pm 1,07\left({ }^{2}\right)\end{aligned}$ \\
\hline
\end{tabular}

(2) Différent de la moyenne obtenue à 6 jours $(\mathrm{P}<0,01)$.

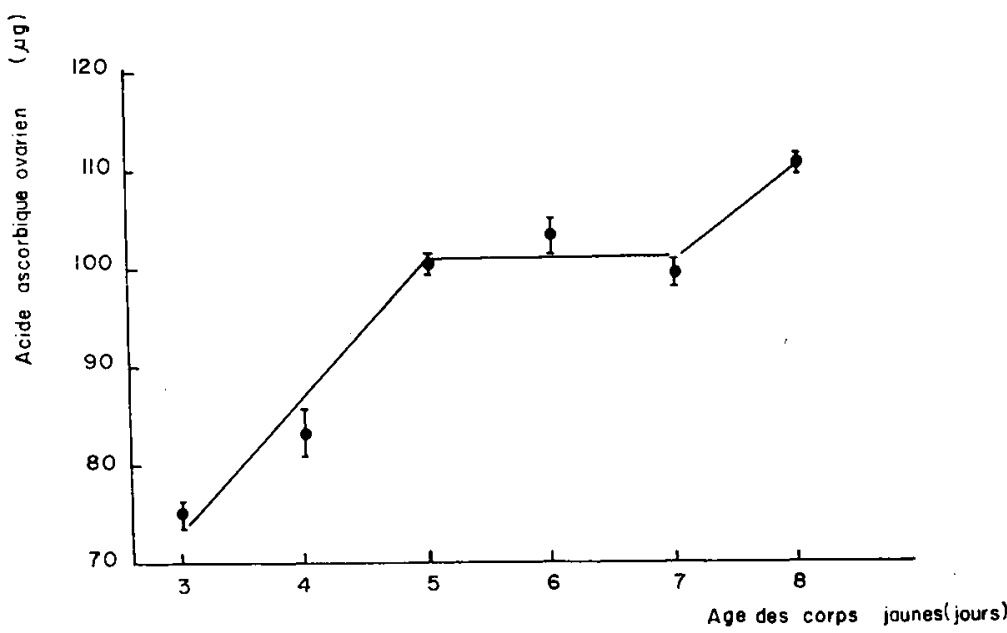

Graphique 4. - Influence de l'âge des corps jaunes sur la quantité d'acide ascorbique contenue dans un ovaire (PMSG 30 U.I., HCG 25 U.I., intervalle entre les injections 62 heures, age des animaux 25 jours.)

par la teneur hypophysaire des rattes réceptrices puisqu'il semble bien que PMSG agisse pour une part en provoquant une décharge des hormones gonadotropes endogènes (WILLIAMS, I945).

Des rattes immatures normales reçoivent l'injection d'hormone sérique, à des âges variant de 2 I jours à 33 jours, tous les animaux étant sevrés à l'âge de 2 I jours (Protocole F, tableau I). Tous ces lots reçoivent l'injection de 25 U.I. d'hormone chorionique $7^{\circ}$ heures plus tard. Les animaux sont abattus cinq jours et demi après cette seconde injection. 
Les résultats sont contenus dans le tableau 6 .

a) Influence de l'âge de l'animal sur le poids ovarien.

La réponse des ovaires en fonction de l'âge est extrêmement nette (graph. 5) : les poids ovariens des animaux âgés de 23 jours lors de l'injection d'hormone sérique sont significativement supérieurs à ceux des animaux ayant reçu cette injection à un âge différent; toutefois la différence entre les poids ovariens des animaux âgés de 23 et de 25 jours est à la limite de la signification.

TABLEAU 6

Influence de l'âge des animaux à l'injection de PMSG sur le poids d'un ovaire $(X)$ et la quantité d'acide ascorbique de cet ovaire $(Y)$

\begin{tabular}{|c|c|c|c|}
\hline $\begin{array}{c}\text { Age } \\
\text { (jours) }\end{array}$ & $\begin{array}{l}\text { Nbre de } \\
\text { données }\end{array}$ & $\bar{X} \underset{(\mathrm{m} g)}{ \pm} \mathrm{Sm}$ & 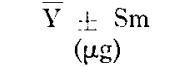 \\
\hline $21 \ldots \ldots \ldots$ & 19 & $78,31=3,38$ & $76,02 \pm 4,68\left({ }^{2}\right)$ \\
\hline $23 \ldots$ & 19 & $92,77 \pm-3,46$ & $97,45 \pm 3,98$ \\
\hline 25 & 19 & $84,8^{\prime} \pm 2,8^{\prime}$ & $77,41 \pm 2,87\left(^{2}\right)$ \\
\hline $27 \ldots$ & 19 & $73,52+2,91\left(^{2}\right)$ & $58,97 \pm 3,13\left({ }^{2}\right)$ \\
\hline 30 & 19 & $68,62 \pm 3,61\left(^{2}\right)$ & $51,58 \pm 4,03\left(^{2}\right)$ \\
\hline 33 & 19 & $62,1^{\prime} \pm 5,64(2)$ & $39,92 \pm 3,40\left({ }^{2}\right)$ \\
\hline
\end{tabular}

$\left(^{2}\right)$ Différent de la moyenne obtenue à 23 jours $(\mathrm{P}<0,01)$

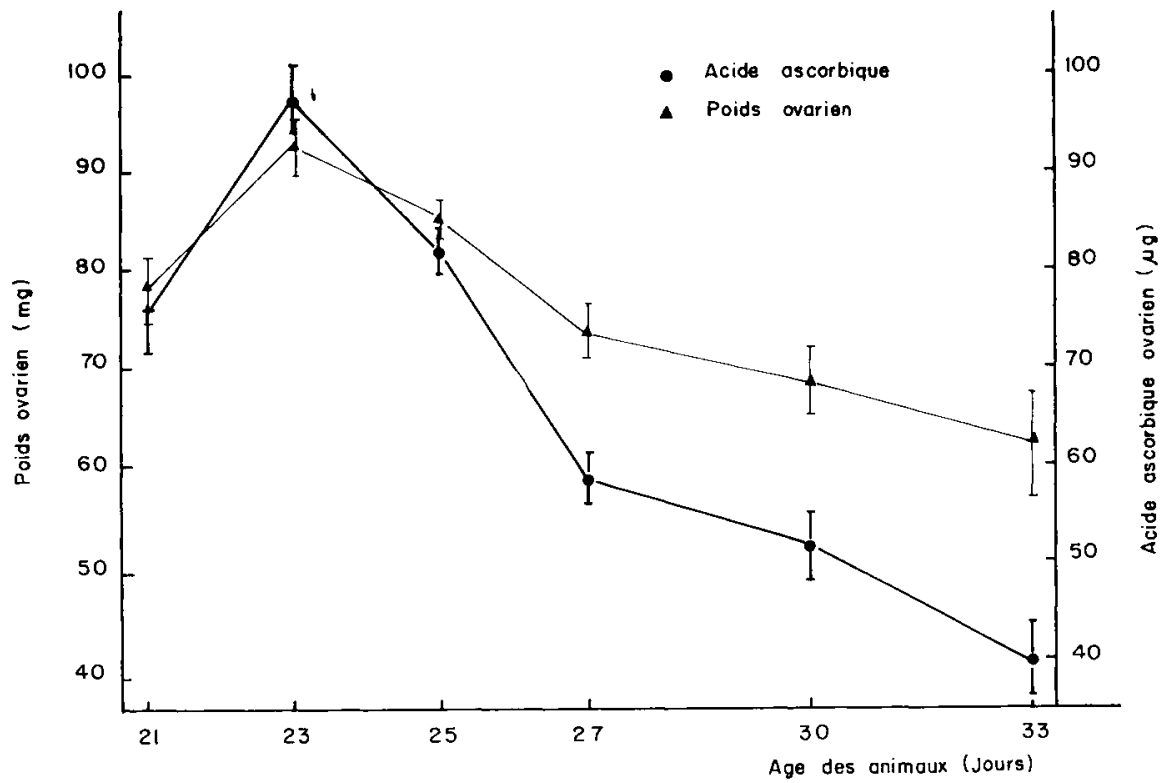

GrapHique 5. - Influence de l'âge des animaux lors de l'injecion de PMSG sur le poids d'un ovaire et son contenu en acide ascorbiyue. (PMSG 30 U.I., HCG 25 U.I., intervalle entre les injections 70 hetures, intervalle entre l'injection de HCG et l'abattage des animaux 5 jours I/2.) 
b) Infuence de l'âge sur l'acide ascorbique ovarien (graph. 5).

Elle est encore plus importante : la teneur en acide ascorbique des ovaires des animaux injectés à 23 jours diffère significativement $(\mathrm{P}<0,0 \mathrm{I})$ de celle des ovaires des animaux injectés à $2 \mathrm{I}$ et 25 jours. En outre au fur et à mesure que l'âge des rattes augmente au-delà de 23 jours, la teneur des ovaires devient beaucoup plus faible que le poids ovarien ne le laisserait supposer. Le traitement par l'hormone sérique doit donc être effectué à 23 jours et dans le choix des animaux, 1'âge semble plus important que le poids : en effet le calcul du coefficient de corrélation poids corporel-poids ovarien, après l'injection de PMSG et de HCG révèle une corrélation extrêmement faible :

$r:+$ o,Ior $(n=204)$ pour des animaux âgés de 23 à 25 jours.

\section{ÉTUDE DU DOSAGE DE ICSH}

Avec les nouvelles conditions de prétraitement des animaux (Protocole standard, tableau I), différents paramètres du dosage de ICSH proprement dit ont été examinés.

\section{Io) Infuence du temps après l'injection de ICSH} sur la diminution de la quantité d'acide ascorbique ovarien

A cet effet $I \mu g$ de LH-NIH est administré à tous les animaux; l'abattage, limitant le temps d'action de l'hormone, est effectué de I à 6 heures après l'injection.

Les résultats (tableau 7 , graph. 6) révèlent que l'acide ascorbique ovarien passe par un minimum 3 heures après l'injection de LH-NIH alors que la variance reste faible. Le retour à la valeur initiale apparaît rapide et le respect d'un protocole défini est indispensable.

\section{TABLEAU 7}

Décroissance de l'acide ascorbique ovarien $(Y)$ en fonction du temps (heures) après l'injection d'hormone interstitielle stimulante

\begin{tabular}{|c|c|c|}
\hline $\begin{array}{l}\text { Heures après } \\
\text { l'injection de ICSIH }\end{array}$ & $\begin{array}{c}\text { Nombre de } \\
\text { données }\end{array}$ & $\widehat{Y}^{*} \pm \operatorname{Sm}(\mu g)$ \\
\hline Contrôles & 59 & $91,7 \pm 1,77$ \\
\hline $\begin{array}{l}1 \ldots \ldots \ldots \ldots \\
2 \ldots \ldots \ldots \ldots \\
3 \ldots \ldots \ldots \\
4 \ldots \ldots \ldots \\
5 \ldots \ldots \ldots \\
6 \ldots \ldots \ldots\end{array}$ & $\begin{array}{l}10 \\
10 \\
10 \\
10 \\
10 \\
10\end{array}$ & $\begin{array}{l}71,5 \pm 2,72 \\
65,9 \pm 1,93 \\
63,5 \pm 2,61 \\
71,8 \pm 3,60 \\
72,3 \pm 3,43 \\
83,0 \pm 6,07\left({ }^{2}\right)\end{array}$ \\
\hline
\end{tabular}




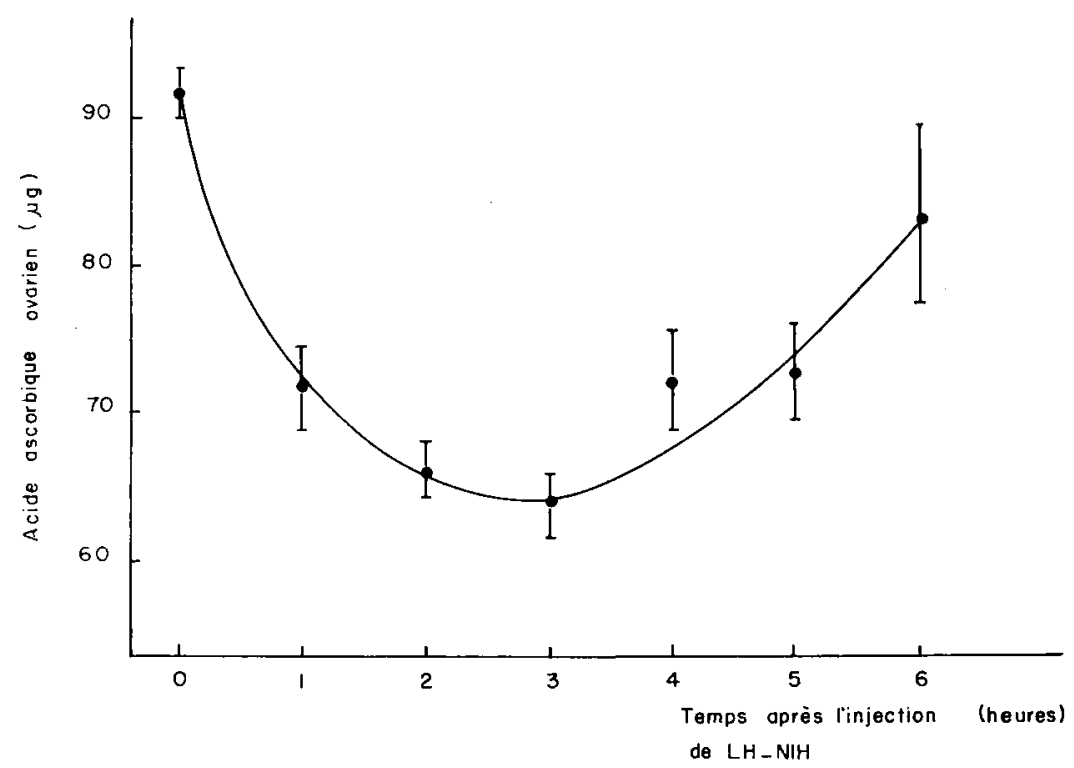

Grapitote 6. - Variation de la quantité d'acide ascorbique contenue dans an onaire en fonction du temps apres linjection de 1 ug de LH-IIH ( $P . M S G$ so U.I., HCG 25 U.I., interalle entre les injections 70 heures, age des animam 23 jours, intervalle entre l'injection de $I C C G$ et l'abattage $6 \mathrm{I} / 2$ jours.)

$2^{\circ}$ V Variation de la quantité d'acide ascorbique en fonction de la dose de LH-NIH injectée

a) Sensibilité.

La sensibilité de la méthode permet de déceler une quantité aussi faible que $0, \mathrm{I} \mu \mathrm{g}$ LH-NIH. Pour $0,2 \mu \mathrm{g}$, la diminution de l'acide ascorbique ovarien est hautement significative.

b) Régression de l'acide ascorbique ovarien en fonction de la dose de LH-NIH.

Des rattes immatures ayant subi le prétraitement standard reçoivent des doses croissantes en progression arithmétique variant de $0, \mathrm{I} \mu \mathrm{g}$ à $6,4 \mu \mathrm{g}$ de $\mathrm{LH}$ NIH.

Les teneurs en acide ascorbique ovarien sont exprimées après correction due au poids (tableau 8). La formule de la droite de régression en coordonnées semilogarithmiques est $\hat{\mathrm{V}}=-9,27 \mathrm{X}+109,48$ (graph. 7) et la linéarité a été trouvée hautement significative.

En pratique nous avons choisi les deux doses de $0,4 \mu \mathrm{g}$ et $\mathrm{I}, 6 \mu \mathrm{g}$ situées au milieu de la portion linéaire de la courbe, qui formeront la droite de référence lors des dosages individuels. 
TABLEAU 8

Diminution de l'acide ascorbique ovarien en fonction de la dose de $\mathrm{LH}-\mathrm{NIH}$

\begin{tabular}{|c|c|c|}
\hline Dose LH-NIH $(\mu g)$ & $\begin{array}{l}\text { Nombre de } \\
\text { données }\end{array}$ & $\begin{array}{c}\text { Acide ascorbique } \\
\text { ovarien } \bar{Y} \pm \operatorname{Sm}(\mu g)\end{array}$ \\
\hline Contrôles & 105 & $113,2 \pm 1,76$ \\
\hline $0,1 \ldots \ldots$ & 15 & $109,48 \pm 4,05$ \\
\hline $0,2 \ldots$ & 15 & $97,54 \pm 4,29$ \\
\hline $0,4 \ldots \ldots \ldots \ldots$ & 15 & $94,98 \pm 5,85$ \\
\hline $0,8 \ldots \ldots \ldots$ & 15 & $84,59 \pm 5,28$ \\
\hline $1,6 \ldots$ & 15 & $71,92 \pm 4,14$ \\
\hline $3,2 \ldots$ & 15 & $58,77 \pm 3,72$ \\
\hline 6,4 & 15 & $55,47 \pm 3,42$ \\
\hline
\end{tabular}

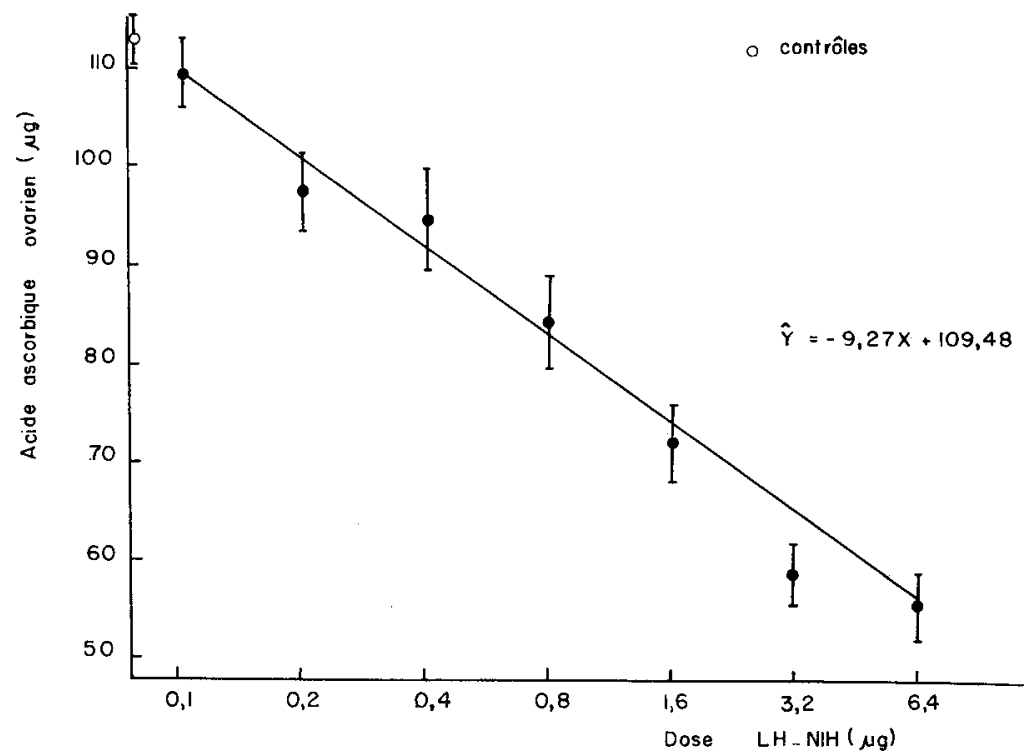

Graphioue 7. - Diminution de la quantité d'acide ascorbique contenue dans un ovaire en tonction du logarithme de la dose de I.H-NIH injectée par voie veineuse (PMSG 30 U.I., HCG 25 U.I., intervalle entre les injections 70 heures, âge des animaux 23 jours, intervalle entre l'injection de HC $G$ et l'abatiage des animaux $6 \mathrm{I} / 2$ jours.)

$\left.3^{\circ}\right)$ Différences génétiques entre plusieurs souches de rats vis-à-vis de l'hormone interstitielle stimulante

Les différences relevées entre plusieurs races de rats vis-à-vis du dosage de ICSH posent le problème de la variabilité à l'intérieur même de l'une d'entre elles.

Trois souches appartenant à la race Wistar, 03, o4 et I2 ont été utilisées pour comparer leur sensibilité à deux doses de $0,4 \mu \mathrm{g}$ et I,6 $6 \mathrm{~g}$ de LH-NIH. 
Le tableau 9 montre que ces trois souches diffèrent déjà d'une façon très importante par leur réponse ovarienne au prétraitement effectué dans les conditions standard définies ci-dessus.

$$
\text { TABLEAU } 9
$$

Infuence de la souche des rattes réceptrices vis-à-vis du prétraitement

\begin{tabular}{c|c|r|r}
\hline Souche & $\begin{array}{c}\text { Nombre } \\
\text { d'animaux }\end{array}$ & $\begin{array}{c}\text { Poids ovarien } \\
\pm \text { Sm }(\mathrm{mg})\end{array}$ & $\begin{array}{c}\text { Acide ascor- } \\
\text { bique } \pm \mathrm{Sm} \\
(\mu \mathrm{g})\end{array}$ \\
\hline $03 \ldots \ldots \ldots \ldots$ & 37 & $101,9 \pm 2,78$ & $121,9 \pm 4,24$ \\
$04 \ldots \ldots \ldots \ldots$. & 37 & $94,1 \pm 3,42$ & $101,6 \pm 5,16$ \\
$12 \ldots \ldots \ldots \ldots$ & 37 & $57,7 \pm 2,10$ & $69,9 \pm 3,46$ \\
\hline
\end{tabular}

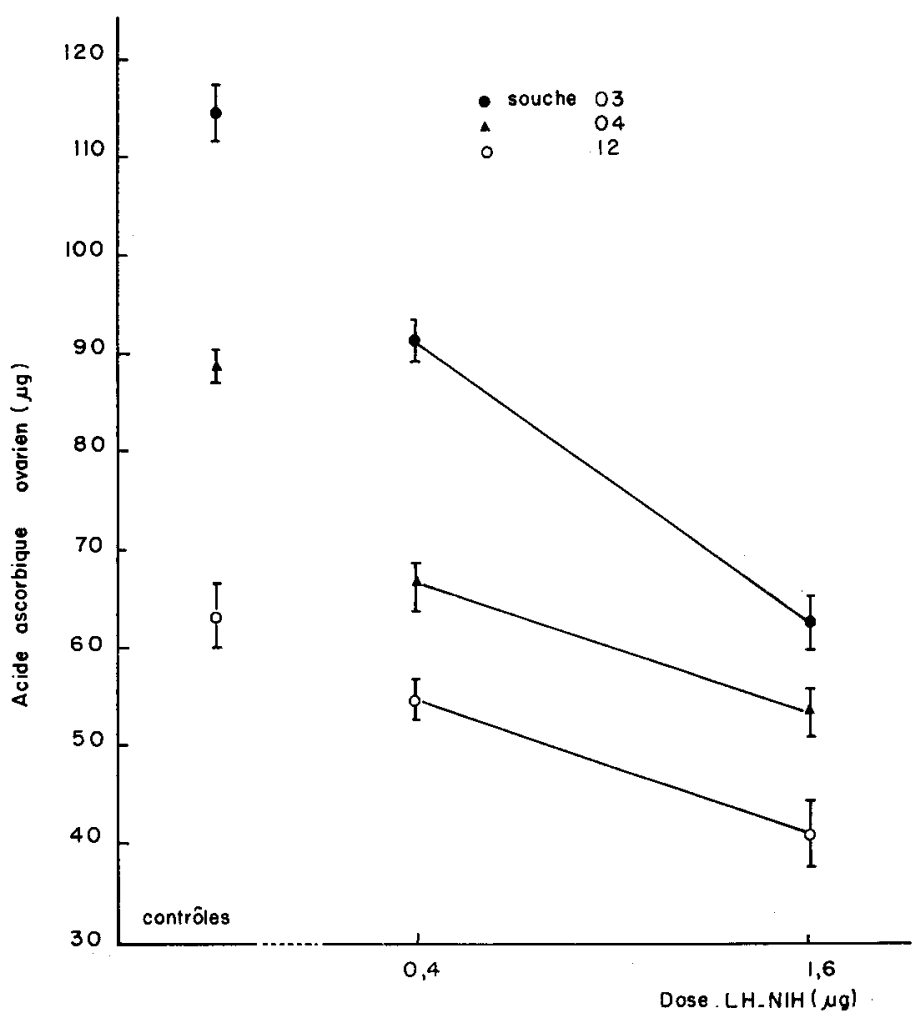

GRAPIIQUE 8. - Comparaison des variations de la quantité d'acide ascorbique dans un ovaire après injection intraveineuse de LH-NIH chez trois souches de Rats Wistar (PMSG 30 U.I., HCG 25 U.I., intervalle entre les injections 70 heures, intervalle entre l'injection de HCG el l'abattage des animaux $6 \mathrm{I} / 2$ jours, agge des animaux 23 jours.)

En outre l'analyse statistique des résultats présentés dans le tableau ro (graph. 8) indiquent que la souche 03 diffère d'une façon très hautement significative des souches 04 et I 2 tant par la teneur en acide ascorbique que par la pente de la droite 
de régression qui est supérieure. La variance étant sensiblement égale dans les trois souches, l'index de précision (GADDUm, I953) est donc nettement le meilleur dans le cas de la souche 03 où il est égal à 0,228 . Cet index serait égal à 0,372 si les trois souches étaient combinées (PEL, ETIER, r963).

TABLEAU IO

Sensibilité à l'hormone interstitielle stimulante en fonction de la souche de rats

\begin{tabular}{|c|c|c|c|c|c|}
\hline Souche & ${ }^{\text {Dose }} \underset{(\mu \mathrm{g})}{\mathrm{LH}-\mathrm{NIH}}$ & $\begin{array}{c}\text { Nombre } \\
\text { d'animaux }\end{array}$ & $\begin{array}{c}\text { Acide ascorbique ovarien } \\
\pm \mathrm{Sm}(\mu \mathrm{g})\end{array}$ & $\begin{array}{l}\text { Pente des } \\
\text { courbes }(b)\end{array}$ & $\begin{array}{l}\text { Index de } \\
\text { précision }(\lambda)\end{array}$ \\
\hline 02 & $\begin{array}{c}\text { Contrôles } \\
0,4 \\
1,6\end{array}$ & $\begin{array}{l}36 \\
18 \\
18\end{array}$ & $\begin{array}{r}114,4 \pm 2,93 \\
91,3 \pm 2,14 \\
62,3 \pm 3,03\end{array}$ & 29,0 & 0,228 \\
\hline $0^{\prime}$ & $\begin{array}{c}\text { Contrôles } \\
0,4 \\
1,6\end{array}$ & $\begin{array}{l}36 \\
18 \\
18\end{array}$ & $\begin{array}{l}88,6 \pm 1,97 \\
66,3 \pm 2,59 \\
53,1 \pm 2,70\end{array}$ & 13,2 & 0,532 \\
\hline 12 & $\begin{array}{c}\text { Contrôles } \\
0,4 \\
1,6\end{array}$ & $\begin{array}{l}36 \\
18 \\
18\end{array}$ & $\begin{array}{l}63,3 \pm 3,30 \\
54,6 \pm 2,16 \\
40,5 \pm 3,83\end{array}$ & 14,1 & 0,529 \\
\hline
\end{tabular}

\section{DISCUSSION}

En modifiant les conditions expérimentales du prétraitement des rattes dans le test de PARLOW, nous avons augmenté considérablement le poids ovarien et la teneur totale en acide ascorbique dans le cas d'animaux de la race Wistar. Ces modifications comprennent une diminution de la dose de PMSG de 50 U.I. à 30 U.I., un allongement de l'intervalle entre les injections de PMSG et de $\mathrm{HCG}$, tel que cet intervalle soit de 70 heures (ou éventuellement de 78 heures, mais dans tous les cas il est indispensable pour des conditions opératoires identiques que ce laps de temps soit conservé constant) et l'utilisation des animaux à un âge plus précoce : 23 jours. En outre, les animaux prétraités sont utilisés seulement entre le $5^{\mathrm{e}}$ et le $7^{\mathrm{e}}$ jour inclus après l'injection de $\mathrm{HCG}$.

Il est remarquable que pour des conditions expérimentales voisines, RowLANDS et WILLIAMS (I944) provoquaient le maximum d'œufs pondus après superovulation chez la Ratte immature à l'aide des deux hormones sérique et chorionique. En particulier, d'après ces auteurs, le nombre d'œufs retrouvés dans les trompes dépendaient essentiellement de la dose de PMSG, avec un maximum pour 30 U.I., la dose de HCG au-dessus d'une valeur-seuil, aux environs de I5 U.I., étant sans influence. Les changements apportés au prétraitement des animaux par PARLOW (I96I) comprennent comme dans notre cas une diminution de la dose de PMSG et l'utilisation des rattes à un âge plus précoce, mais ces modifications se révèlent insuffisantes pour nos souches d'animaux et les conditions expérimentales dégagées 
de cette étude permettent encore une augmentation significative de la teneur totale de 1'acide ascorbique ovarien. Il en résulte que le coefficient de corrélation liant le poids de l'ovaire et l'acide ascorbique ovarien augmente lui-même de $+0,60$ à + 0,90 , ce qui revient à réduire considérablement l'erreur par analyse de covariance : en effet, la correction de l'acide ascorbique en fonction du poids ovarien diminue les variations non contrôlées de plus de 80 p. roo au lieu de 36 p. Ioo seulement.

Par ailleurs une étude comparative sur plusieurs souches de rattes (non publiée) indique que la pente de la droite de régression de l'acide ascorbique en fonction de la dose ICSH est d'autant meilleure que la teneur initiale en vitamine C est plus forte. En agissant ainsi sur la pente et l'erreur, l'index de précision a été diminué de 0,4 à 0,2 au moins, permettant une étude quantitative correcte.

Les résultats concernant la sensibilité et la zone de linéarité de la droite de régression, sont très voisins de ceux obtenus par Mc CANN et TAL EISNIK (Ig6o) et PARLOW (I96I) sur les races Holtzman et Sherman et par SCHMIT'T-EILMENDORFF et LoRaine (Ig62), Robertson et Hutchinson (I962) sur la race Wistar. De même que ceux de Goldstern (Ig6I) et SchMidT-EilmendorfF et LoRAine (I962), nos résultats indiquent que la diminution de l'acide ascorbique dans l'ovaire est maximale 3 heures après l'injection intraveineuse de ICSH, la variabilité restant faible pour cet intervalle de temps.

En définitive, s'il semble bien y avoir une différence génétique entre les races de rats utilisées dans les laboratoires, la race Holtzman paraissant la plus favorable, des modifications du protocole du prétraitement permettent cependant l'emploi des animaux de race Wistar dans les conditions expérimentales convenables pour le dosage d'ICSH.

Reçu pour publication en juin 1963 .

SUMMARY

A CRITICAL STUdY OF ICSH DOSAGE BY OVARIAN ASCORBIC ACID DEPLETION (PARLOW'S TEST)

Difficulties in the use of the determination of ICSH by the PARLow technique (Ovarian Ascorbic Acid Depletion) have been encountered when the test-animals belong to the Wistar race (ScHMIDT ElMENDORFF and LORAINe, I962 ; ROBERTSON and HuTCHINSON, I962). This study shows that the modifications of the pretreatment of animals of the Wistar race considerably increase the precision of the method by increasing the ovarian ascorbic acid.

In comparison with the PARLOW procedure (196I) these modifications include :

- the use of younger animals right from the start of the pre-treatment : 23 days. This age corresponds to the maximum sensitivity to the seric hormone;

- a decrease in the dose of PMSG (30 U.I.), nevertheless the seric hormone must be tested periodically as ageing brings about a decrease in activity;

- a bigger interval between the injections of PMSG and HCG ( $70 \mathrm{~h}$ or even $78 \mathrm{~h}$ ); the interval must be kept constant.

Moreover the precision of the method is still further increased by the use of an interval of $3 \mathrm{~h}$ between the injection of ICSH and the killing of the animals on one hand and by the genetic selection within the Wistar race.

These modifications together make the use of Wistar rats suitable for experimental purposes. 


\section{RÉFÉRENCES BIBLIOGRAPHIQUES}

BLIss C. I., I952. The statistics of bioassav, with special reference to the vilamins. Acad. Press, New York.

Claesson L., Hillarp N. A., Högberg B., Hökfelt B., I949. Changes in the ascorbic acid content in the interstitial gland of the rabbit ovary following gonadotrophic stimulation. Acta endocr., Copenhagen, $2,249-256$.

Emmens C. W., I948. Principles of biological assay. Chapman and Hall, Iondon.

Gaddum J. H., I953. Bioassays and mathematics. Pharmacol. Rev., 5, 87-I34.

Goldstein D. P., Sturgis S. H., I96r. Luteinizing hormone induced depletion of ascorbic acid in the rat ovary. Amer. J. Physiol., 201, I053-1056.

KARG H., 1958. Ascorbinsäuredynamik in ovar als gonadotropinachweis. Klin. Wschr., 35, 643.

MC CANn S. M., TALEISNik S., ig6o. Effect of luteinizing hormone and vasopressin on ovarian ascorbic acid. Amer. J. Phyiol., 199, 847-850.

Mindlin R. L., Butler A. M., 1937. The determination of ascorbic acid in plasma. J. Biol. Chem., 122, $67.3-686$.

Munson P. L., communication persomelle de Parlow.

Parlow A. F., i958. A rapid biassay method for LH and factors stimulating LH secretion. Feder. Proc. 17, 402 .

PARLow A. F., I961. Human pituitary gonadolrophins. 300-310 A. Albert, Springfield, Thomas.

Pelletier J., i963. Étude de l'hormone stimulant l'interstitielle : Méthode de dosage et variation de la teneur hypophysaire du Bélier soumis à différentes durées d'éclairement. Thèse de $3^{\mathrm{c}}$ cvcle, Faculté des Sciences, Paris

Robertson H. A., ILtcinsson J. S. M., ig62. The levels of FSH and LH in the pituitary of the ewe in relation to follicular growth and ovulation. J. Endocrin., 24, I43-15I.

Rowlands I. W., I944. The production of ovulation in the immature rat. J. Endocrin., 3, 384-391.

SChmidt-LilmendorfF H., Loraine J. A., I962. Some observations on th: ovarian ascorbic acid depletion method in rats as a test for luteinizing hormone activity. J. Endocrin., 23, 41 3-42 I.

Williams P. C., 1945. Studies of the biological action of serum gonadotrophin. 3-role of endogenous gonadotrophin. J. Endocrin., 4, I37-I42. 